Sawtooth superlattices in a two-band semiconductor

This article has been downloaded from IOPscience. Please scroll down to see the full text article.

1994 Semicond. Sci. Technol. 91358

(http://iopscience.iop.org/0268-1242/9/7/010)

View the table of contents for this issue, or go to the journal homepage for more

Download details:

IP Address: 147.96.14.16

The article was downloaded on 22/02/2013 at 17:27

Please note that terms and conditions apply. 


\title{
Sawtooth superlattices in a two-band semiconductor
}

\author{
F Domínguez-Adame and $B$ Méndez \\ Departamento de Física de Materiales, Universidad Complutense, 28040 Madrid, \\ Spain
}

Received 14 March 1994, accepted for publication 15 April 1994

\begin{abstract}
We consider electron dynamics in two-band semiconductor superlattices within the envelope function approximation. We develop a numerical method, based on the properties of the periodic continued fractions, to find the dispersion relation inside allowed minibands and to obtain the envelope functions. As an application, we concentrate on GaAs sawtooth-doped superiattices, consisting of periodic alternating $n$ - and $p$-type $\delta$-doped sheets separated by undoped material. Results are compared with one-band semiconductor predictions, and we find that coupling of bands in the host semiconductor indeed gives rise to relevant effects, especially for higher minibands.
\end{abstract}

\section{Introduction}

In recent years heterostructures and superlattices have been intensively investigated as a source of novel physical properties as well as for device applications. Recently, attention has been paid to sawtooth-doped superlattices, which consist of periodic alternating $\mathrm{n}$ - and p-type $\delta$-doped sheets separated by undoped material [1, 2]. Such doping profiles are generated by interrupting the crystal growth of the host material and evaporating the doping impurity during molecular beam epitaxy. Under appropriate growth conditions, excellent confinement of dopant atoms can be achieved and diffussion of impurities is irrelevant [2]. As a consequence, the doping profile along the growth direction $z$ can be represented as

$N(z)=N_{\mathrm{D}}^{2 \mathrm{D}} \sum_{j} \delta(z-j L)-N_{\mathrm{A}}^{2 \mathrm{D}} \sum_{j} \delta(z-j L-L / 2)$

where $N_{\mathrm{D}}^{2 \mathrm{D}}$ and $N_{\mathrm{A}}^{2 \mathrm{D}}$ are the two-dimensional donor and acceptor concentrations, and $L$ is the period of the superlattice. Usually samples are compensated so that $N_{\mathrm{D}}^{2 \mathrm{D}}=N_{\mathrm{A}}^{2 \mathrm{D}}=N^{2 \mathrm{D}}$. The chain of alternating $\delta$ doped sheets results in a sawtooth modulation of both conduction and valence band edges. This sawtooth structure allows us to obtain the largest potential modulation possible on a short length scale; the internal electric field is $F=e N^{2 \mathrm{D}} / 2 \epsilon$, e being the elementary charge and $\epsilon$ the static dielectric constant, and the band-edge modulation is $e F L / 2$. This value is twice the modulation of conventional $(n-i-p-i)$ doping superlattices [2]. The potential in each period of the superlattice is a V-shaped well and carriers can then occupy quantized energy levels due to quantum size effects. Nevertheless, resonant coupling among all identical eigenstates of individual wells leads to the formation of minibands, as occurs in periodically $\mathrm{n}$ type $\delta$-doped semiconductors (see [3-5] and references therein). Hence electronic states are actually extended in perfect superlattices in the absence of external fields.

The analysis of the resulting electronic structure has been carried out by various methods. Most of them are based on the envelope function approximation [6]. Neglecting the non-parabolicity of the bands, the system is described by a scalar Hamiltonian corresponding to decoupled bands. The wave equation is a Schrödingerlike equation for a particle of effective mass $m^{*}$ in a $\mathrm{V}$-shaped potential well. In this case exact solutions are possible since the corresponding Schrödinger equation may be transformed into the Airy differential equation (see [7] and references therein). This isotropic and parabolic conduction-band approximation usually works well in some semiconductors, as is the case for GaAs, but only at moderate electric fields [8]. On the other hand, however, decoupled bands cannot adequately describe narrow-gap semiconductors or those superlattices whose band modulation is comparable to the magnitude of the gap, mainly due to non-parabolicity effects $[9,10]$. The latter situation is found, for instance, in GaAs sawtooth superlattices: although $\mathrm{GaAs}$ is usually regarded as a wide-gap semiconductor, the magnitude of the band modulation for $N^{2 D}=10^{13} \mathrm{~cm}^{-2}$ and $L=20 \mathrm{~nm}$ is found to be $600 \mathrm{meV}$, which is more than $40 \%$ of the gap in GaAs. It is then clear that a more realistic band structure is essential to properly describe the electronic structure of sawtooth superlattices. It is known that twoband models such as we give here represent direct-band compounds quite well [11].

The aim of this paper is twofold. First, we develop a simple numerical method for the determination of the 
dispersion relation inside allowed minibands, the energy of miniband edges and envelope functions in arbitrary superlattices of two-band semiconductors. Besides the periodicity, we require the built-in potential and the position-dependent gap to be symmetric around the centre of the unit cell of the superlattice. Actually, this is not a serious restriction since most semiconductor superlattices present a centre of symmetry. Second, as a specific illustration of the method, we calculate the miniband structure of sawtooth-doped superlattices of GaAs for several growth parameters and compare our results with those obtained by means of the oneband approximation. We shall show that significant differences exist between the two approaches even when the magnitude of the band modulation is not too large. These differences will be discussed in view of the analogy existing between two-band models and the relativistic Dirac theory of electrons.

\section{Model}

We treat the resulting electronic structure in the superlattice by means of the effective-mass $\boldsymbol{k} \cdot \boldsymbol{p}$ approximation. Therefore, the electronic wavefunction is written as a sum of products of band-edge orbitals with slowly varying envelope functions, assuming that the superlattice potential is also slowly varying. This approximation fails if this potential changes appreciably on the scale of superlattice period compared with the gap. However, envelope function results still give a good account of tunnelling experiments where electric fields are as high as $0.1 \mathrm{~V} \mathrm{~nm}^{-1}$ [12]. This value is well above the internal electric fields encountered in sawtooth superlattices of GaAs; for doping densities as high as $N^{2 \mathrm{D}}=10^{13} \mathrm{~cm}^{-2}$ the internal electric field is about $0.03 \mathrm{~V} \mathrm{~nm}^{-1}$. Therefore, the use of the envelope function theory is justified in our case since we never exceed such doping densities.

Keeping only the two nearby bands, there are two coupled envelope functions describing the conductionband and valence-band states of the semiconductor, subject to an effective $2 \times 2$ Dirac-like equation. Assuming that both the gap and the gap centre depend only on $z$, the resulting equation for the envelope functions in the conduction and valence bands can be written as

$$
\begin{gathered}
{\left[\begin{array}{cc}
\frac{1}{2} E_{\mathrm{g}}(z)-E+V(z) & -\mathrm{i} \hbar v \partial \\
-\mathrm{i} \hbar v \partial & -\frac{1}{2} E_{\mathrm{g}}(z)-E+V(z)
\end{array}\right]} \\
\quad \times\left(\begin{array}{c}
f_{\mathrm{c}}(z) \\
f_{\mathrm{v}}(z)
\end{array}\right)=0
\end{gathered}
$$

where $\partial=\mathrm{d} / \mathrm{d} z$. Here $E_{\mathrm{g}}(z)$ is the position-dependent gap and $V(z)$ gives the absolute position of the gap centre. In the case of periodic superlattices of period $L$ both functions are periodic, i.e. $E_{\mathrm{g}}(z+L)=E_{\mathrm{g}}(z)$ and $V(z+L)=V(z)$. The velocity $v$ is related to Kane's momentum matrix elements and is given by $\vec{v}^{2}=E_{\mathrm{g}} / 2 \bar{m}^{*}$. In spite of the fact that both $E_{\mathrm{g}}$ and the effective mass $m^{*}$ are in general position-dependent, the value of $v$ is almost constant in direct-gap III-V semiconductors, reflecting the similarity in the zonecentre Bloch functions [12]. Hereafter we will assume this constancy in the superlattice. It should be mentioned that the non-zero in-plane momentum can be easily absorbed in the parameter definitions, as mentioned in [12], and we will ignore it in the following. Equation (2) can be decoupled in the standard fashion to obtain

$$
\begin{aligned}
\partial^{2} f_{\mathrm{c}}(z) & -\frac{\partial V(z)-\partial E_{\mathrm{g}}(z) / 2}{V(z)-E-E_{\mathrm{g}}(z) / 2} \partial f_{\mathrm{c}}(z) \\
& +\frac{1}{\hbar^{2} v^{2}}\left[(V(z)-E)^{2}-\frac{1}{4} E_{\mathrm{g}}^{2}(z)\right] f_{\mathrm{c}}(z)=0
\end{aligned}
$$

and

$$
f_{\mathrm{v}}(z)=\frac{\mathrm{i} \hbar v}{V(z)-E-E_{\mathrm{g}}(z) / 2} \partial f_{\mathrm{c}}(z) .
$$

Hence one can deal only with $f_{\mathrm{c}}$ since $f_{\mathrm{v}}$ may be found using $(3 b)$.

\section{Numerical method}

There exist, at present, several numerical methods for one-dimensional band structure calculations based on the discretized Schrödinger equation [13, 14], whereas the $2 \times 2$ Dirac equation has received much less attention $[15,16]$. The method we present here is a generalization of previous work by the authors [15] in order to include the spatial dependence of the gap (i.e. the equivalent of relativistic scalar-like potentials in the ordinary Dirac theory). To this end, the properties of continued fractions will be used to find the dispersion relation inside allowed minibands of the superlattice.

Let us divide the unit cell of the superlattice $[-L / 2, L / 2]$ into $N+1$ equal parts of length $s=$ $L /(N+1)$, which defines the grid $z_{n}=n s-L / 2$ $(n=0,1, \ldots, N)$. Using a finite-difference scheme, the discretized form of $(3 a)$ at any point of the grid is given by

$$
\left(1-a_{n}\right) f_{c}\left(z_{n+1}\right)+\left(1+a_{n}\right) f_{c}\left(z_{n-1}\right)-\left(2-b_{n}\right) f_{c}\left(z_{n}\right)=0
$$

where for brevity we have defined

$$
\begin{aligned}
& a_{n}=\frac{1}{4} \frac{V\left(z_{n+1}\right)-V\left(z_{n-1}\right)-E_{\mathrm{g}}\left(z_{n+1}\right) / 2+E_{\mathrm{g}}\left(z_{n-1}\right) / 2}{V\left(z_{n}\right)-E-E_{\mathrm{g}}\left(z_{n}\right) / 2} \\
& b_{n}=\left(\frac{s}{h v}\right)^{2}\left[\left(V\left(z_{n}\right)-E\right)^{2}-\frac{1}{4} E_{\mathrm{g}}^{2}\left(z_{n}\right)\right] .
\end{aligned}
$$

Due to the spatial periodicity of the superlattice, envelope functions must satisfy the Bloch theorem along the growth direction. This implies that $f_{c}(z)=$ $\exp (\mathrm{i} k z) U_{\mathrm{c}}(z)$, with $U_{\mathrm{c}}(z+L)=U_{\mathrm{c}}(z)$ where $k$ is the component of the wavevector along $z$. Note that 
equation $(3 b)$ ensures that $f_{\mathrm{v}}(z)$ is also a Bloch function. If we define

$$
\begin{aligned}
\alpha_{n} & =\frac{1-a_{n}}{1+a_{n}} \\
\beta_{n} & =\frac{2-b_{n}}{1+a_{n}} \\
R_{n} & =\mathrm{e}^{-\mathrm{i} k s} \frac{U_{\mathrm{c}}\left(z_{n}\right)}{U_{\mathrm{c}}\left(z_{n+1}\right)}
\end{aligned}
$$

we find the following continued fraction:

$$
R_{n-1}=\beta_{n}-\alpha_{n} / R_{n}
$$

The periodicity of the function $U_{c}$ gives rise to the periodic boundary condition $R_{0}=R_{N+1}$ for the continued fraction (7). To deal with periodic continued fractions we define the $n$th numerator $A_{n}$ and denominator $B_{n}$ through the relations [17]

$$
\begin{aligned}
& A_{n+1}=\beta_{n+2} A_{n}-\alpha_{n+1} A_{n-1} \\
& B_{n+1}=\beta_{n+2} B_{n}-\alpha_{n+1} B_{n-1}
\end{aligned}
$$

along with the initial values $A_{-1}=B_{0}=1, A_{0}=$ $\beta_{1}$ and $B_{-1}=0$. It is worth mentioning that both $A_{n}$ and $B_{n}$ depend on the energy $E$ but they do not explicitily depend upon any particular value of $k$; this is an important point in obtaining the dispersion relation. Since we are assuming that $V(-z)=V(z)$ and $E_{\mathrm{g}}(-z)=E_{\mathrm{g}}(z)$, it is straightforward although somewhat tedious to demonstrate that the dispersion relation inside allowed minibands is given by (see [15] for further details)

$$
\cos k L=\frac{1}{2}\left[A_{N}(E)-\alpha_{N+1}(E) B_{N-1}(E)\right] .
$$

Note that the right-hand side of (9) is recursively evaluated from (8) at every energy value. Once its absolute value is less than unity, a real value of $k$ is found and the dispersion relation inside allowed minibands $E=E(k)$ is obtained. Conversely, forbidden minigaps appear whenever $\left|A_{N}-\alpha_{N+1} B_{N-1}\right|>2$. In addition, the time-reversal symmetry $E(-k)=E(k)$ is preserved with this numerical technique.

In many physical applications one requires not only the dispersion relation inside allowed minibands but also the wavefunction at some particular energy value $E$. This may be accomplished as follows. The periodicity of the continued fraction (7) allows us to write the equation for $R_{0}$ in terms of $A_{n}$ and $B_{n}$ [15]:

$$
B_{N} R_{0}^{2}-\left(\alpha_{N+1} B_{N-1}+A_{N}\right) R_{0}+\alpha_{N} A_{N-1}=0
$$

and proceeding recursively from (7) one finds every $R_{n}$. Since $k=k(E)$ is known, the function $U_{c}$ may be evaluated, apart from the normalization factor, by means of $(6 c)$. Finally, $f_{c}$ and $f_{\mathrm{v}}$ are obtained in a simple way.

Some words concerning truncation errors are in order. Since the method uses a standard finite-difference scheme, the truncation error is of the order of $s^{2}$. Results may be improved by means of the Richardson extrapolation formula, where the corresponding energy value is given by $\left(4 E_{2 N}-E_{N}\right) / 3, E_{N}$ being the value obtained with $N$ subdivisions of the unit cell. The truncation error is then of the order of $s^{4}$. Moreover, the Numerov scheme, whose error is also of the order of $s^{4}$, may be easily implemented in equation (4) if higher accuracy is required, since it is a three-point rule. However, equation (4) gives rather accurate results, at least within the scope of the present work, even if $s$ is not too small compared with the superlattice period. We should stress that the numerical method is quite general, in the sense that it is valid for a large variety of superlattices in the two-band approximation, assuming the existence of a centre of symmetry. Also, the outlined numerical method can be adapted to one-band semiconductors, i.e. those superlattices whose miniband structure can be properly described by means of an effective-mass Schrödinger equation. This reduction is accomplished simply by taking $a_{n}=0$ and $b_{n}=$ $2 m^{*} s^{2}\left[E-V\left(z_{n}\right)\right] / \hbar^{2}$ in equation (4).

\section{GaAs sawtooth superlattices}

We study the resulting miniband structure in GaAs sawtooth-doping superlattices within the two-band model and compare the obtained results with the oneband model predictions. We consider compensated samples $N_{\mathrm{D}}^{2 \mathrm{D}}=N_{\mathrm{A}}^{2 \mathrm{D}}=N^{2 \mathrm{D}}$ with two-dimensional doping densities ranging from 1 up to $10 \times 10^{12} \mathrm{~cm}^{-2}$. In order to investigate the effects of the superlattice period $L$ on the electronic structure, we have considered samples with $L=10 \mathrm{~nm}$ and $L=15 \mathrm{~nm}$. The value of the gap is assumed to be constant along the whole sample $\left(E_{\mathrm{g}}=1.42 \mathrm{eV}\right)$ and we have taken $\hbar v=0.9 \mathrm{eV} \mathrm{nm}$. The modulation of the conduction and valence bands in each period is then described by the function $V(z)=e F|z|$ with $|z| \leqslant L / 2$. To check the accuracy of the numerical method, the number of grid points in the unit cell varied from 500 to 1000; in both cases we obtained the same results within the desired accuracy.

Figure 1 shows the miniband structure of superlattices with $N^{2 D}=10^{13} \mathrm{~cm}^{-2}$, for $L=10 \mathrm{~nm}$ and $L=15 \mathrm{~nm}$. Similar results are found for other doping concentrations. Energies are measured from the bottom of the V-shaped potential well in both cases. The modulations of the conduction band are $290 \mathrm{meV}$ and $430 \mathrm{meV}$ respectively. As expected, lower bands are almost dispersionless due to the small overlap of neighbouring identical states. This effect is more apparent on increasing the superlattice period. Figure 1 also shows results corresponding to the one-band model, obtained by solving the effective-mass Schrödinger equation. A detailed analysis of the obtained data indicates that lower minibands are practically independent of the model used (one-band or two-band). The energy of the lower, almost non-dispersive miniband is about $130 \mathrm{meV}$, in excellent agreement with the groundstate energy obtained by directly solving the Schrödinger equation for an infinite $V$-shaped potential well, given by 


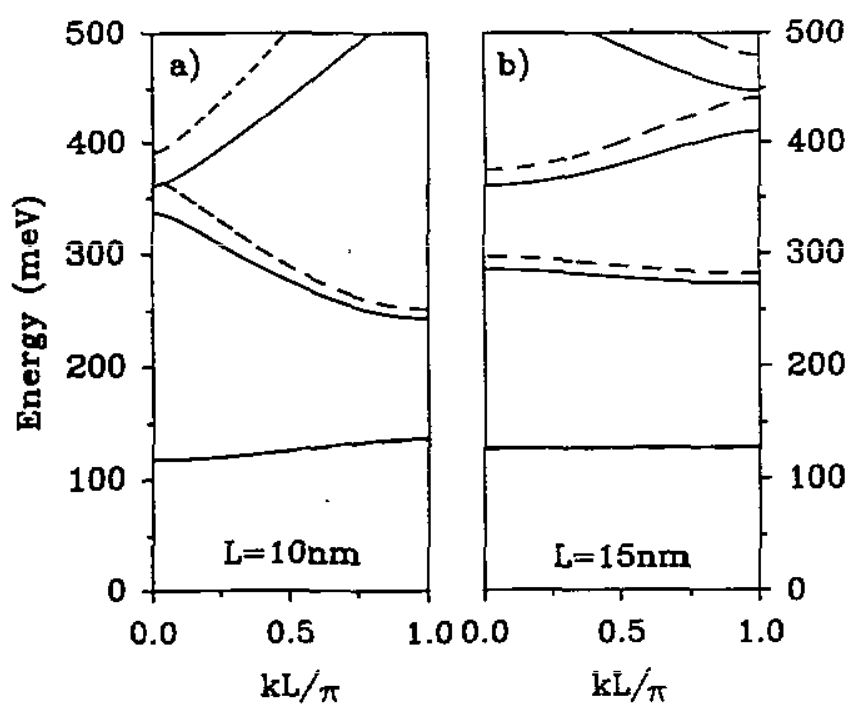

Figure 1. Miniband structures in GaAs sawtooth superiattices for $N^{2 D}=10^{13} \mathrm{~cm}^{-2}$ and superlattice period (a) $L=10 \mathrm{~nm}$ and (b) $L=15 \mathrm{~nm}$, for two-band (full curves) and one-band (broken curves) models.

$1.02\left(\hbar^{2} e^{2} F^{2} / 2 m^{*}\right)^{1 / 3} \simeq 126 \mathrm{meV}$ [2]. On increasing the energy, however, the two models yield different dispersion relations because non-parabolicity effects become increasingly important. This fact implies that coupling of bulk bands becomes more important at higher energy, and that the one-band model is meaningful only in the case of the ground-state miniband. One can observe that the widths of both minigaps and allowed minibands decrease in the case of the two-band model in comparison with one-band predictions. This fact is independent of the doping concentration.

Figure 2 shows the widths of the first two lower minibands and the minigap between the two, as functions of the doping concentration for a period of $L=10 \mathrm{~nm}$. Since modulation of band edges increases linearly with doping concentration, the V-shaped potential being deeper in the gap, the minigap width becomes larger whereas the miniband widths decrease in both models. Notice that two-band predictions are always smaller than one-band results in the whole range of doping studied in this work. As mentioned above, the lower miniband is almost unaffected by the coupling of bulk bands; this result is clearly seen in figure $2(a)$. On the other hand. the difference between the minigaps in the two models increases slightly with increasing doping concentration, as observed in figure $2(b)$. However, the width of the second miniband decreases linearly with the same slope in the two models, as shown in figure $2(c)$, and thus the difference is independent of doping concentration in the range considered, being of the order of $25 \mathrm{meV}$.

Let us comment that the shrinkage of the electronic spectrum seems to be a universal property of Dirac-like equations. It was earlier observed in Dirac-KronigPenney models [18], in connection with relativistic electron dynamics in solids, but it is not restricted to this simple case. The energy spectrum also shrinks in more elaborate models of solids, such as the relativistic Mathieu potential [15] and DiracKronig-Penney models with non-local potentials [19].
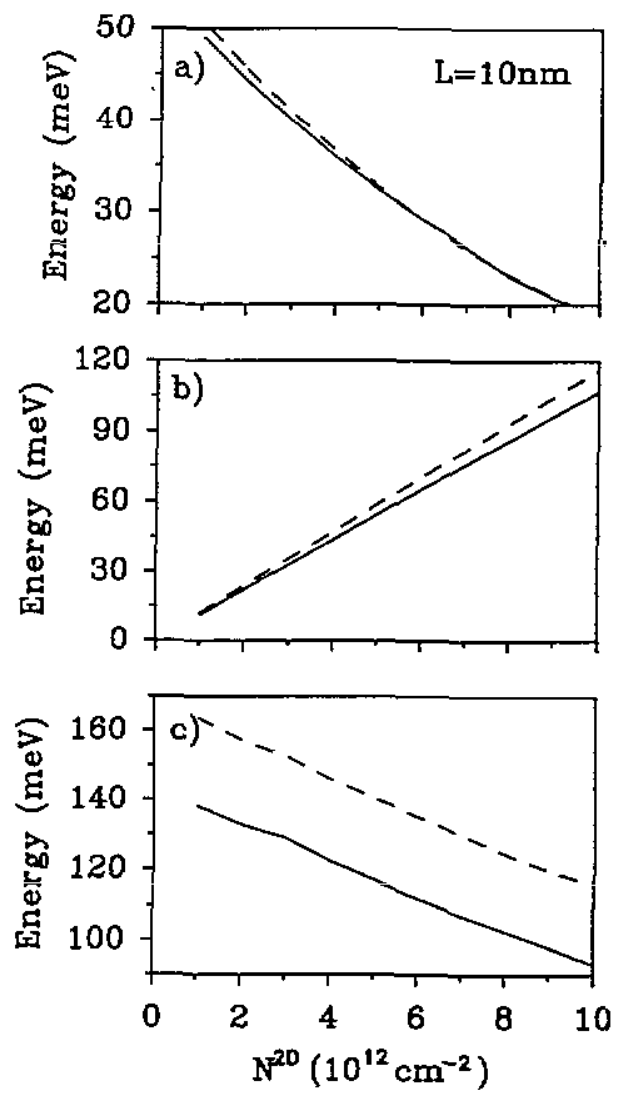

Figure 2. Widths of (a) the first miniband, (b) the first minigap and (c) the second miniband as functions of the doping concentration for a superlattice period $L=10 \mathrm{~nm}$ for two-band (full curves) and one-band (broken curves) models.

Moreover, the periodicity of the potential is not an essential condition for observing this effect; for instance, similar results are found in Dirac-KronigPenney models on quasiperiodic superlattices [20]. Therefore, we may conclude that one of the main results we have found in sawtooth superlattices when a two-band model is used, namely the reduction of the widths of minibands and minigaps, may probably be observed in other kinds of superlattice. These effects should be more dramatic on decreasing the gap of the material, when coupling of bands becomes increasingly important. We feel that it is important to mention here that, to our knowledge, the shrinkage of the electronic spectrum has been previously discussed only with regard to relativistic effects in solids. The fact that the restmass energy of electrons ( $\mathrm{MeV}$ ) is very much larger than typical values of electron energy (eV) makes it hard to investigate relativistic effects in solids experimentally. In contrast, we suggest that two-band semiconductor superlattices are well suited to testing the validity of 'relativistic' predictions, as occuirs in the case of the spectrum shrinkage described in this work, due to the formal analogy between two-band models and the Dirac equation. The situation would then be similar to what happened with the well-known Stark-Wannier ladders: they were predicted to appear in actual solids but their existence has been firmly established only in quantumwell superlattices [21]. 


\section{Summary}

By making use of the algebraic properties of periodic continued fractions, we are able to develop a simple numerical scheme to find the dispersion relation inside allowed minibands in periodic superlattices of two-band semiconductors, whose electron dynamics is described by means of an effective Dirac equation. To illustrate the proposed numerical method, we have focused our attention on GaAs sawtooth-doping superlattices, consisting of periodic alternating n- and p-type $\delta$ doped sheets separated by undoped GaAs. We have found that two-band and one-band models give different miniband structures in GaAs sawtooth superlattices, even when GaAs is usually not considered as a narrowgap semiconductor and conduction and valence band modulation is not large (the largest value we have studied in this work is only $30 \%$ of the GaAs bulk gap). One of the more relevant features is that the twoband model leads to a shrinkage of the whole electronic spectrum, this effect being more apparent with increasing energy. In particular, we found that the shrinkage of the second miniband is independent of doping concentration, although conduction band modulation increases linearly with doping. We believe that this reduction should be observable experimentally since it amounts between $10 \%$ and $20 \%$ of the miniband width.

\section{Acknowledgments}

The authors would like to thank E Maciá and A Sánchez for a critical reading of the manuscript. This work is partially supported by UCM under Project PR161/934811.

\section{References}

[1] Schubert E F, Horikoshi Y and Ploog K 1985 Phys. Rev. B 321085

[2] Schubert E F, Ullrich B, Harris T D and Cunningham J E 1988 Phys. Rev. B 388305

[3] Chico L, García-Moliner F and Velasco V R 1993 Phys. Rev. B 4811427

[4] Domínguez-Adame F, Méndez B and Maciá E 1994 Semicond. Sci. Technol. 9263

[5] Méndez B and Domínguez-Adame F 1994 Phys. Rev. B 4911471

[6] Bastard G 1981 Phys. Rev. B 245693

[7] Ahn D 1993 Phys. Rev. B 487981

[8] Hagon J P, Jaros M and Herbert D C 1989 Phys. Rev. B 406420

[9] Zawadski W 1983 J. Phys. C: Solid State Phys. 16229

[10] Bastard G 1989 Wave Mechanics Applied to Semiconductor Heterostructures (Paris: Editions de Physique)

[11] Callaway J 1991 Quantum Theory of the Solid State (New York: Academic) p 36

[12] Beresford R 1993 Semicond. Sci. Technol. 81957

[13] Vigneron J P and Lambin Ph 1979 J. Phys. A: Math. Gen. 121961

[14] Killingbeck J 1980 J. Phys. A: Math. Gen. 13 L35

[15] Méndez B and Domínguez-Adame F 1991 J. Phys. A: Math. Gen. 24 L331

[16] Méndez B, Domínguez-Adarne F and Maciá E 1993 J. Phys. A: Math. Gen. 26171

[17] Wall H S 1967 Analytic Theory of Continued Fractions (New York: Chelsea) p 35

[18] Glasser M L and Davison S G 1970 Int. J. Quantum Chem. IIIS p 867

[19] Domínguez-Adame F and González M A 1992 Physica B 176180

[20] Domínguez-Adame F and Sánchez A 1991 Phys. Lett. 159A 153

[21] Agulló-Rueda F, Méndez E E and Hong J M 1989 Phys. Rev. B 401357 